\title{
A conceptual model of compensation/decompensation in lumbar segmental instability
}

\author{
T. Barz ${ }^{\mathrm{a}, 1}$, M. Melloh ${ }^{\mathrm{b}, \mathrm{c}, *, 1}$, S.J. Lord ${ }^{\mathrm{d}, \mathrm{e}}$, R. Kasch ${ }^{\mathrm{f}}$, H.R. Merk ${ }^{\mathrm{g}}$, L.P. Staub ${ }^{\mathrm{h}}$ \\ a Department of Orthopaedic Surgery, Asklepios Klinikum Uckermark, Schwedt/Oder, Germany \\ ${ }^{\mathrm{b}}$ Centre for Health Sciences, School of Health Professions, Zurich University of Applied Sciences, Winterthur, Switzerland \\ ${ }^{\mathrm{C}}$ Centre for Medical Research, University of Western Australia, Nedlands, Australia \\ ${ }^{\mathrm{d}}$ NHMRC Clinical Trials Centre, University of Sydney, Sydney, NSW, Australia \\ ${ }^{\mathrm{e}}$ Epidemiology Er Medical Statistics, School of Medicine, University of Notre Dame, Sydney, NSW, Australia \\ ${ }^{\mathrm{f}}$ Clinic and Outpatient Clinic for Orthopedics and Orthopedic Surgery, University Medicine Greifswald, Greifswald, Germany \\ ${ }^{\mathrm{g}}$ Department of Orthopaedic Surgery, University Medicine Greifswald, Germany \\ ${ }^{\mathrm{h}}$ Institute for Evaluative Research in Orthopaedic Surgery, University of Bern, Switzerland
}

\section{A R T I C L E I N F O}

\section{Article history:}

Received 11 November 2013

Accepted 4 June 2014

\begin{abstract}
A B S T R A C T
Lumbar spinal instability (LSI) is a common spinal disorder and can be associated with substantial disability. The concept of defining clinically relevant classifications of disease or 'target condition' is used in diagnostic research. Applying this concept to LSI we hypothesize that a set of clinical and radiological criteria can be developed to identify patients with this target condition who are at high risk of 'irreversible' decompensated LSI for whom surgery becomes the treatment of choice. In LSI, structural deterioration of the lumbar disc initiates a degenerative cascade of segmental instability. Over time, radiographic signs become visible: traction spurs, facet joint degeneration, misalignment, stenosis, olisthesis and de novo scoliosis. Ligaments, joint capsules, local and distant musculature are the functional elements of the lumbar motion segment. Influenced by non-functional factors, these functional elements allow a compensation of degeneration of the motion segment. Compensation may happen on each step of the degenerative cascade but cannot reverse it. However, compensation of LSI may lead to an alleviation or resolution of clinical symptoms. In return, the target condition of decompensation of LSI may cause the new occurrence of symptoms and pain. Functional compensation and decompensation are subject to numerous factors that can change which makes estimation of an individual's long-term prognosis difficult. Compensation and decompensation may influence radiographic signs of degeneration, e.g. the degree of misalignment and segmental angulation caused by LSI is influenced by the tonus of the local musculature. This conceptual model of compensation/decompensation may help solve the debate on functional and psychosocial factors that influence low back pain and to establish a new definition of non-specific low back pain. Individual differences of identical structural disorders could be explained by compensated or decompensated LSI leading to changes in clinical symptoms and pain. Future spine surgery will have to carefully define and measure functional aspects of LSI, e.g. to identify a point of no return where multidisciplinary interventions do not allow a re-compensation and surgery becomes the treatment of choice.
\end{abstract}

(c) 2014 Elsevier Ltd. All rights reserved.

\section{Introduction}

Lumbar spinal instability (LSI) is a common spinal disorder and can be associated with substantial disability. Management of LSI is

\footnotetext{
Source of support in form of grants: none.

* Corresponding author at: Centre for Health Sciences, School of Health Professions, Zurich University of Applied Sciences, Technikumstrasse 71, 8401 Winterthur, Switzerland. Tel.: +41589344396.

E-mail address: markus.melloh@zhaw.ch (M. Melloh).

${ }^{1}$ Both authors contributed equally.
}

challenging because the clinical and radiological criteria used for diagnosis are not yet clearly defined. Moreover, the existing diagnostic criteria do not perform well to distinguish between patients who are likely to benefit from conservative management and those who will not improve without surgical intervention. This has led to considerable uncertainty and wide variation in the selection of patients for surgery.

The concept of defining clinically relevant classifications of disease or 'target condition' is used in diagnostic research [1]. It is used to develop medical tests and cut-off points that can better 
Table 1

Definitions of lumbar segment instability.

- Reduced resistance to movement (Zhao et al., 2005)

- Ratio "neutral zone"/range of motion (Mimura et al., 1994)

- Increase "neutral zone", or physiological limits (Panjabi, 1992)

- Condition in which a small load applied to a system causes a marked displacement (Ashton-Miller, 1991)

- Loss of the spine's ability to maintain patterns of displacement under physiologic loads (White and Panjabi, 1990)

- Radiological retro-displacement of a vertebra during flexion (Knutsson, 1944)

- "Pseudo-spondylolisthesis" (Schmorl and Junghanns, 1932)

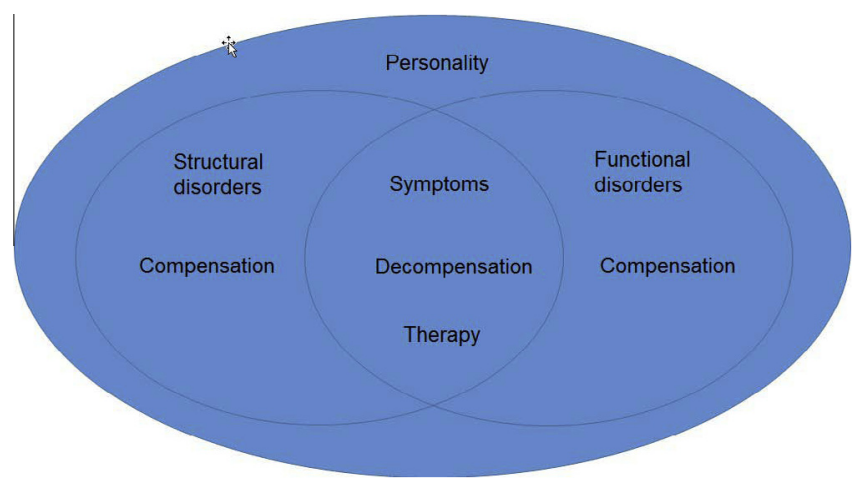

Fig. 1. Overlap of structural and functional lumbar segment instability. Adapted from Tsuij H: Comprehensive Atlas of Lumbar Spine Surgery. Mosby Year Book, St Louis; 1990 [61].

inform clinical decisions, for example, the development of tests for staging patients with cancer to help discriminate between those at low versus high risk of disease recurrence to guide decisions for adjuvant chemotherapy. It is also used when evaluating tests, to design test accuracy studies to provide measures of test performance that are relevant to the decisions the test will be used to inform in clinical practice [1].

In this paper, we propose that the concept of defining the target condition to guide the development and evaluation of medical tests can be extended to improve clinical practice for conditions such as lumbar spinal degeneration, where more clinically meaningful definitions of disease are needed to guide treatment decisions. We discuss two of the most common degenerative disorders of the lumbar spine: structural or functional lumbar segmental instability, and lumbar spinal stenosis (LSS). For both conditions no consensus exists on the definition using clinical, radiological and pathological criteria (Table 1, Fig. 1) [2-7].

\section{Hypothesis}

Applying the concept of the target condition to lumbar spinal degeneration, we define the more severe spectrum of disease where symptom alleviation is unlikely to occur without surgical intervention as the target condition most relevant for clinical decisions. We hypothesize that a set of clinical and radiological criteria can be developed to identify patients with this target condition who are at high risk of 'irreversible' decompensated LSI for whom surgery becomes the treatment of choice. These criteria will be extremely valuable to distinguish between a 'compensated' condition where the patient is likely to remain in a compensated state of the disease - benefitting from conservative treatment; and a 'decompensated' condition where the patient has reached the point of no return into decompensation - requiring surgical treatment

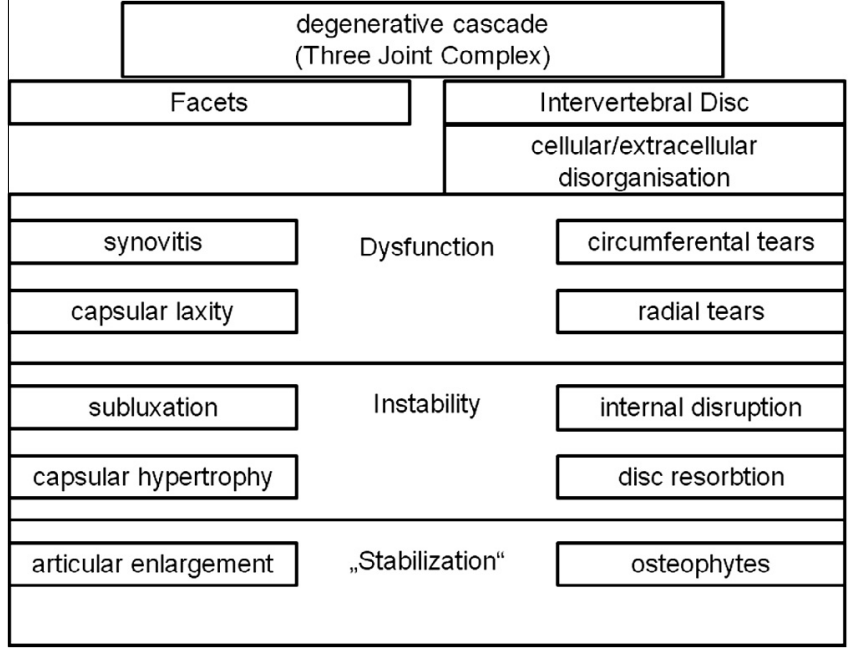

Fig. 2. Degeneration of lumbar disc, i.e. degenerative cascade. Adapted from Kirkaldy-Willis WH and Bernard TN. Managing Low Back Pain. Elsevier Ltd., Oxford; 4th edition 1999 [62].

\section{Current concept of spinal degeneration}

The current understanding of degeneration of the spine is based on the concept of a degenerative cascade of anatomical structures within the lumbar motion segment, which comprises soft tissue (ligaments, joint capsules, local and distant musculature, intervertebral discs) and bony elements (two adjacent vertebral bodies, facet joints) [8-10] (Fig. 2).

In the lumbar motion segment model as introduced by Junghanns, the spinal segments are organized in a way that they permit individual movement [11]. Intervertebral discs, anterior and posterior longitudinal ligaments, facet joints, ligamentum flavum, parts of the vertebral body between the processus spinosus and transversus, and muscles interplay with one another for normal function. When a disorder or injury occurs, the whole motion segment is affected and the degenerative cascade begins which can lead to the symptoms and signs of LSI (and LSS).

\section{A new conceptual model of compensation/decompensation in lumbar spinal degeneration}

Given the poor correlation between clinical and radiographic variables for the diagnosis of LSI [12-15], a binary model of compensation/decompensation - where a period of compensation is followed by a single shift to decompensation - is likely to be an oversimplification of the natural history of LSI. Therefore, we propose a new conceptual model of compensation/decompensation that incorporates both aspects to reflect the more complex relationship between clinical signs and radiological findings that is observed in practice as outlined below.

Evidence demonstrating the association between electromyography (EMG) measured muscle activation and risk of low back pain (LBP) $[16,17]$ suggests the functional elements of the motion segment are also influenced by non-functional factors such as psychosocial factors. The functional elements allow, within different individual limits, changes in function and position of the motion segment, and consequently, a compensation of the degeneration of the motion segment [18-20] (Table 2). They also influence the shape of the load/range of motion curve [21,22]. Compensation may happen on each step of the degenerative cascade but cannot reverse it. However, on each step, compensation 
Table 2

Influencing psychosocial factors on lumbar motion segment.

Mental health
Depression
Somatization
Fear-avoidance beliefs
Pain catastrophizing
Optimism/resilience
Marital/relationship status
Educational status
Social support

Table 3

Definition of compensation, adaptation, and decompensation according to M. Forte. Ewen B. [Osteopathic procedures and osteopathic medicine. A manual medicine osteopathic career in chain reactions]. Manuelle Med. 2013;51:291-294 [60].

Compensation
Physiological reaction of a body part on functional or structural variation of
another body part
Adaptation
Adaptation of tissues to maintain functional compensation
Decompensation
Imbalance of a body part caused by a conflict between at least 2 different
mechanisms of compensation or adaptation

of LSI may lead to an alleviation or resolution of clinical symptoms. The functional elements of the motion segment are organized in a cybernetic model where the command variable may change (e.g. pain, function, and pressure relief of the nerve). It remains unclear whether a potential overload of this model might lead to degeneration per se. In return, if compensation is no longer possible, a distinct new target condition is reached: decompensation of LSI which may cause the new occurrence of clinical symptoms and pain [23-25]. The different individual limits in which compensation takes place decrease over time and are dependent upon overload of the functional elements and progression of degeneration.

In osteopathic and manual medicine the term compensation is used in a similar way. In contrast, decompensation is understood as conflict of opposing mechanisms of functional compensation or structural adaptation (Table 3 ).
Table 4

Influencing genetic/physiological factors of degenerative cascade.

Segmental spinal mobility
Gait
Unequal leg length
Body mass index
Serum lipid levels
Electric activity of skeletal muscles
Muscle strength
Cross-sectional area of muscles
Pelvic incidence
Pelvic tilt and sacral slope

Table 5

Radiographic signs of lumbar segment instability.

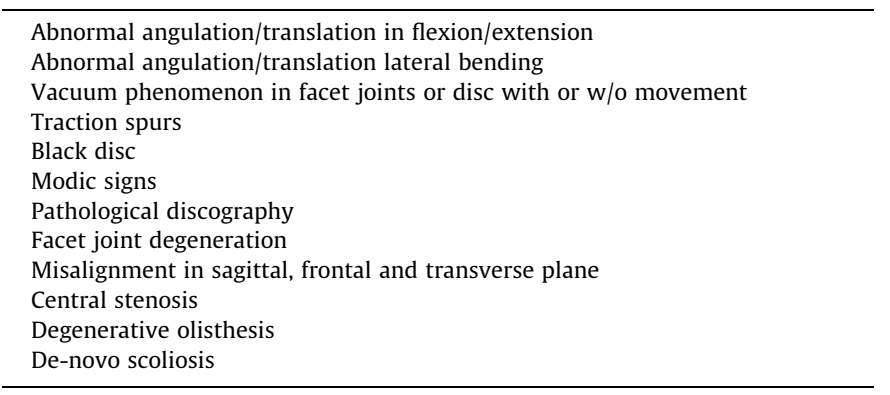

Structural deterioration of the lumbar disc - influenced by genetic [26-28] and physiological factors - leads to lumbar misalignment in coronal and sagittal balance and consequently, to the loss of lumbar lordosis [29-31] (Table 4). This initiates a degenerative cascade of LSI also leading to LSS [32,33]. Over time radiographic signs become visible such as traction spurs, facet joint degeneration, misalignment, stenosis, olisthesis and de novo scoliosis [34-36] (Table 5).

Diagnostic and management decisions in LSI are often made on the grounds of expert opinions focusing on symptoms and imaging. However, as clinical and radiographic information poorly correlates, there is an apparent missed opportunity between the daily clinical practice of diagnosing patients with back disorders and

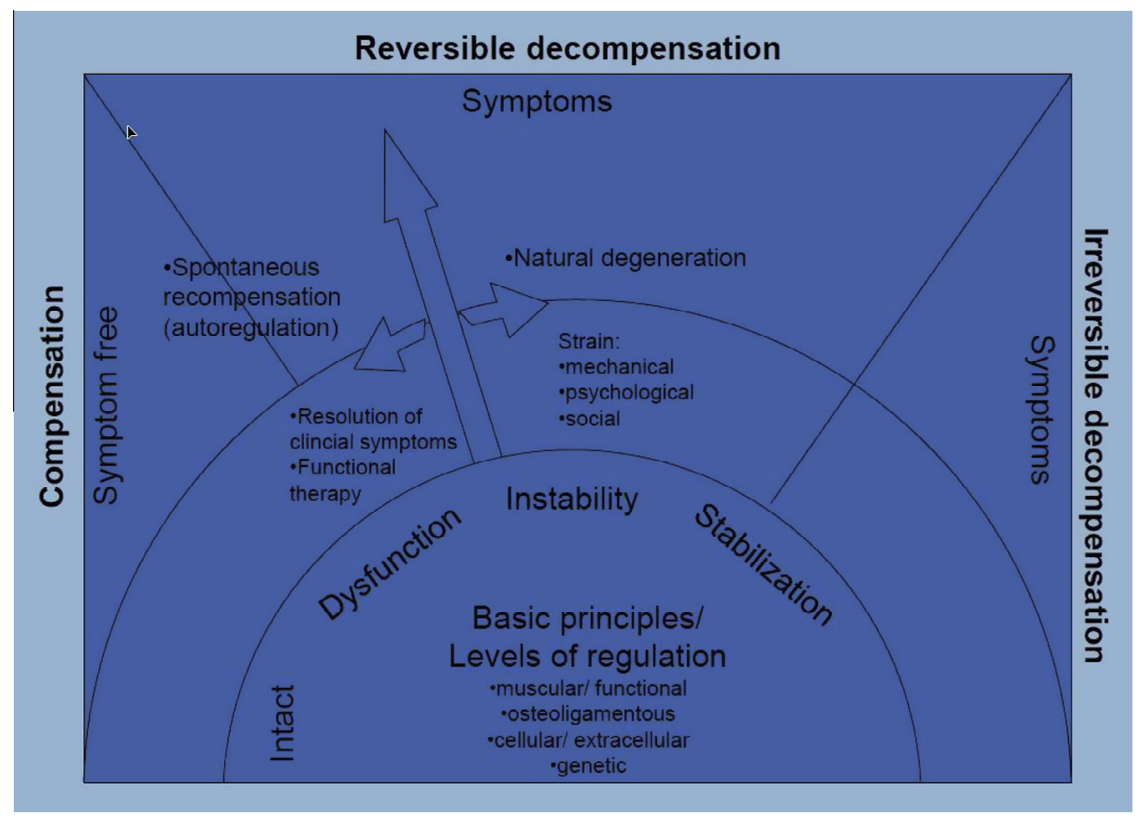

Fig. 3. Model of compensation/decompensation. 
the scientific evidence available from systematically gathering and interpreting data from groups of similar patients. Furthermore, a thorough holistic approach when assessing patients with LSI including physiological and psychological factors is rarely taken into consideration.

There is a need for an extended pathophysiological/psychosocial concept of compensation/decompensation in LSI (Fig. 3) [3740]. Functional compensation and decompensation are subject to numerous factors that can vary between individuals and change over time which results in a poor correlation between radiographic degenerative signs and clinical symptoms. This makes estimation of an individual's prognosis of the benefit of surgical or nonsurgical treatment difficult [41]. In the following a broader conceptual framework of compensation/decompensation and examples will be presented that may help solve the debate on the prognostic value of functional and psychosocial factors of influence.

Compensation and decompensation may influence some nonstructural radiographic signs of degeneration, e.g. the degree of misalignment and segmental angulation caused by LSI is influenced by the tonus of the local musculature $[42,43]$. Factors which might influence whether a patient with LSI, LSS or lumbar misalignment will be symptomatic, i.e. be in the state of decompensation, are different electric activities of skeletal muscles, muscle strength [44-47], cross-sectional area of muscles and pelvic parameters $[30,31,48]$.

Conflicting results are presented in the literature regarding the influence of muscle activity on patients with LSI, LSS or lumbar misalignment. This could be due to the fact that different individual spine curves require different individual stabilizing musculature [49-54].

This conceptual model of compensation/decompensation can be extended to include LSS as one example of its application beyond LSI. Consider an 88-years-old patient with symptoms for 8 weeks and dysfunction with claudication and walking distance of $40 \mathrm{~m}$ due to mechanical strain and natural degeneration, with images illustrating degenerative stenosis and instability due to segmental kyphosis, leading to the condition of spontaneous recompensation and resolution of clinical symptoms without functional therapy (Fig. 3). Patients with early compensated LSS usually present with LBP as an initial symptom. At a later stage, leg fatigue, pain, numbness and weakness of the lower extremities commence. These symptoms begin or worsen with the onset of walking or by standing upright and are promptly relieved when leaning forward, sitting or lying down.

As another example, consider a female patient aged 69 with symptoms for 6 months and dysfunction with claudication and no pain free walking distance due to mechanical and psychosocial strain, with images demonstrating degenerative stenosis and instability due to a facet cyst and secondary lumbar kyphosis, resulting in irreversible decompensation and no resolution of clinical symptoms despite functional therapy. Once symptoms of neurogenic intermittent claudication that occurs during walking and forces patients to rest are present the condition of decompensated state has usually been reached. In radiological terms decompensation could be expressed by a positive Nerve Root Sedimentation Sign where nerve roots are tethered at the level of the stenosis $[55,56]$. It is assumed that similar to the degenerative cascade in LSI there is a 'narrowing' cascade in LSS whose determining factors are still poorly understood, as illustrated by common over and underdiagnosis of LSS $[57,58]$.

\section{Discussion}

In this paper we propose that the diagnostic research concept of defining clinically meaningful target conditions can be extended to
Table 6

Current multidisciplinary interventions.

Multidisciplinary biopsychosocial rehabilitation

Rehabilitation programs

Functional restoration programs

Back schools

Work hardening

Conditioning exercises

address the challenging problem of selecting treatments for patients with lumbar spinal degeneration. We hypothesize that additional clinical and radiological criteria can be developed to define a target condition of decompensated spinal degenerative disease to identify patients who have reached the point where there is only a very low probability of returning to a compensated state. These criteria will be enormously valuable to identify patients at high risk of 'irreversible' decompensated LSI/LSS for whom surgery becomes the treatment of choice.

To test this hypothesis, we suggest further research is needed to identify the criteria that can be used to define the decompensated target condition. Future studies need to identify predictors that can help discriminate between patients at low and high probability of remaining in an irreversibly decompensated state. This will require prognostic studies, i.e. data collected from clinical studies of patients managed with conservative care, whereby the potential radiological, physiological and psychological predictors are compared with the clinical outcome (symptom improvement). The two types of studies that can provide data to identify the optimal clinical and test criteria or cut-points to define the presence of the target condition for treatment decisions are: (i) cohort studies that can be used to examine the relationship between clinical factors, test results and subsequent clinical outcomes (prognosis) to develop risk groups that can inform decisions about whether treatment is indicated; and (ii) treatment trials that can be used to identify patient subgroups (defined by clinical factors and test results) where the benefits of treatment outweigh the harms.

Finally, this conceptual model of compensation/decompensation may be extended to spinal disorders in general and beyond. It could help solve the debate on the proportion of influence of functional and psychosocial factors on LBP and to establish a new definition of non-specific LBP. Individual differences could be explained by compensated or decompensated LSI leading to changes in clinical symptoms and pain. As long as identically structural characteristics, such as translation in ante and retroflexion for LSI or the area of the dural sac in MRI for LSS, are employed it is not surprising that these characteristics are of only limited predictive value for the presence of clinical symptoms.

\section{Conclusions}

This model of compensation/decompensation aims to solve contradictions in existing concepts in the spine field by explaining why identical structures may lead to varying symptoms. Future spine surgery will have to carefully define and measure functional aspects of LSI. This will have to include the identification of specific signs for a point of no return where multidisciplinary interventions do not allow a re-compensation and surgery becomes the treatment of choice [59] (Table 6). Knowledge of signs for a point of no return, i.e. a status of decompensation is crucial for decision-making in today's health care systems that are scarce of resources.

\section{Conflict of interest statement}

None to declare. 


\section{References}

[1] Lord SJ et al. Target practice: choosing target conditions for test accuracy studies that are relevant to clinical practice. BMJ 2011;343:d4684.

[2] Amundsen $\mathrm{T}$ et al. Lumbar spinal stenosis. Clinical and radiologic features. Spine 1995;20(10):1178-86.

[3] Barz T et al. The diagnostic value of a treadmill test in predicting lumbar spinal stenosis. Eur Spine J 2008;17(5):686-90.

[4] Chiodo A et al. Magnetic resonance imaging vs. electrodiagnostic root compromise in lumbar spinal stenosis: a masked controlled study. Am J Phys Med Rehabil 2008;87(10):789-97.

[5] Kanno H et al. Dynamic change of dural sac cross-sectional area in axial loaded magnetic resonance imaging correlates with the severity of clinical symptoms in patients with lumbar spinal canal stenosis. Spine (Phila Pa 1976) 2012;37(3):207-13.

[6] Konno $S$ et al. Development of a clinical diagnosis support tool to identify patients with lumbar spinal stenosis. Eur Spine J 2007;16(11):1951-7.

[7] Sirvanci $M$ et al. Degenerative lumbar spinal stenosis: correlation with oswestry disability index and MR imaging. Eur Spine J 2008;17(5):679-85.

[8] Haig AJ. Paraspinal denervation and the spinal degenerative cascade. Spine J 2002;2(5):372-80.

[9] O'Sullivan PB et al. Lumbar repositioning deficit in a specific low back pain population. Spine 2003;28(10):1074-9.

[10] Kirkaldy-Willis WH. The pathology and pathogenesis of low back pain. In: Managing low back pain. New York, NY: Churchill Livingstone; 1988. p. 49.

[11] Junghanns H. Functional pathology of the intervertebral disks as a basis of clinical observations. Langenbecks Arch Klin Chir Ver Dtsch Z Chir 1951;267:393-417.

[12] Chou $\mathrm{R}$ et al. Imaging strategies for low-back pain: systematic review and meta-analysis. Lancet 2009;373(9662):463-72.

[13] Hasegawa $\mathrm{K}$ et al. Evaluation of lumbar segmental instability in degenerative diseases by using a new intraoperative measurement system. J Neurosurg Spine 2008;8(3):255-62.

[14] Modic MT, Ross JS. Lumbar degenerative disk disease. Radiology $2007 ; 245(1): 43-61$.

[15] Takayanagi $\mathrm{K}$ et al. Using cineradiography for continuous dynamic-motion analysis of the lumbar spine. Spine (Phila Pa 1976) 2001;26(17):1858-65.

[16] Goel VK et al. A combined finite element and optimization investigation of lumbar spine mechanics with and without muscles. Spine 1993;18(11): 1531-41.

[17] Heydari A et al. EMG analysis of lumbar paraspinal muscles as a predictor of the risk of low-back pain. Eur Spine J 2010;19(7):1145-52.

[18] Janowski K, Steuden S, Kurylowicz J. Factors accounting for psychosocial functioning in patients with low back pain. Eur Spine J 2010;19(4):613-23.

[19] Ramond A et al. Psychosocial risk factors for chronic low back pain in primary care - a systematic review. Fam Pract 2011;28(1):12-21.

[20] Gatchel RJ, Polatin PB, Mayer TG. The dominant role of psychosocial risk factors in the development of chronic low back pain disability. Spine 1995;20(24):2702-9.

[21] Berthonnaud E et al. Analysis of the sagittal balance of the spine and pelvis using shape and orientation parameters. J Spinal Disord Tech 2005;18(1): 40-7.

[22] Barrey $C$ et al. Sagittal balance of the pelvis-spine complex and lumbar degenerative diseases. A comparative study about 85 cases. Eur Spine J 2007;16(9):1459-67.

[23] van Hooff ML et al. Daily functioning and self-management in patients with chronic low back pain after an intensive cognitive behavioral programme for pain management. Eur Spine J 2010;19(9):1517-26.

[24] Mayer $\mathrm{F}$ et al. The intensity and effects of strength training in the elderly. Dtsch Arztebl Int 2011;108(21):359-64.

[25] Imagama $S$ et al. Back muscle strength and spinal mobility are predictors of quality of life in middle-aged and elderly males. Eur Spine J 2011;20(6):954-61.

[26] Urano $T$ et al. Single-nucleotide polymorphism in the hyaluronan and proteoglycan link protein 1 (HAPLN1) gene is associated with spinal osteophyte formation and disc degeneration in Japanese women. Eur Spine J $2011 ; 20(4): 572-7$.

[27] Kalb $S$ et al. Genetics of the degenerated intervertebral disc. World Neurosurg 2012;77(3-4):491-501.

[28] Battie MC, Videman T, Parent E. Lumbar disc degeneration: epidemiology and genetic influences. Spine (Phila Pa 1976) 2004;29(23):2679-90.

[29] Lewit K, Janda V. The development of articulation disorders of the spine in childhood and the principles of preventing vertebragenic disorders. Hippokrates 1963;34:308-16.

[30] Le Huec JC et al. Sagittal imbalance cascade for simple degenerative spine and consequences: algorithm of decision for appropriate treatment. Eur Spine J 2011;20(Suppl. 5):699-703.

[31] Roussouly P, Pinheiro-Franco JL. Biomechanical analysis of the spino-pelvic organization and adaptation in pathology. Eur Spine J 2011;20(Suppl. 5):609-18.
[32] Iguchi T et al. Intimate relationship between instability and degenerative signs at L4/5 segment examined by flexion-extension radiography. Eur Spine J 2011;20(8):1349-54.

[33] Samartzis D et al. A population-based study of juvenile disc degeneration and its association with overweight and obesity, low back pain, and diminished functional status. J Bone Joint Surg Am 2011;93(7):662-70.

[34] Butler D et al. Discs degenerate before facets. Spine 1990;15(2):111-3.

[35] Hadjipavlou AG et al. The pathophysiology of disc degeneration: a critical review. J Bone Joint Surg Br 2008;90(10):1261-70.

[36] Albert HB et al. The prevalence of MRI-defined spinal pathoanatomies and their association with modic changes in individuals seeking care for low back pain. Eur Spine J 2011;20(8):1355-62.

[37] Freemont AJ. The cellular pathobiology of the degenerate intervertebral disc and discogenic back pain. Rheumatology (Oxford) 2009;48(1):5-10.

[38] Yoon ST, Patel NM. Molecular therapy of the intervertebral disc. Eur Spine J 2006;15(Suppl. 3):S379-88.

[39] Chan D et al. Genetics of disc degeneration. Eur Spine J 2006;15(Suppl. 3):S317-25.

[40] Neidlinger-Wilke $C$ et al. Regulation of gene expression in intervertebral disc cells by low and high hydrostatic pressure. Eur Spine J 2006;15(Suppl. 3):S372-8.

[41] Scale D, Zichner L. Natural course in lumbar disk prolapse. Orthopade 1994;23(3):236-42.

[42] Imagama $S$ et al. Influence of sagittal balance and physical ability associated with exercise on quality of life in middle-aged and elderly people. Arch Osteoporos 2011;6(1-2):13-20.

[43] Butler HL et al. Directed attention alters the temporal activation patterns of back extensors during trunk flexion-extension in individuals with chronic low back pain. Eur Spine J 2010;19(9):1508-16.

[44] Macedo LG et al. Graded activity and graded exposure for persistent nonspecific low back pain: a systematic review. Phys Ther 2010;90(6):860-79.

[45] Macedo LG et al. Effect of motor control exercises versus graded activity in patients with chronic nonspecific low back pain: a randomized controlled trial. Phys Ther 2012;92(3):363-77.

[46] Johanson E et al. The effect of acute back muscle fatigue on postural control strategy in people with and without recurrent low back pain. Eur Spine J 2011;20(12):2152-9.

[47] Kong WZ et al. Effects of muscle dysfunction on lumbar spine mechanics. A finite element study based on a two motion segments model. Spine (Phila Pa 1976) 1996;21(19):2197-206 [discussion 2206-7].

[48] Barrey C et al. Sagittal balance disorders in severe degenerative spine. Can we identify the compensatory mechanisms? Eur Spine J 2011;20(Suppl. 5):626-33.

[49] $\mathrm{Hu} \mathrm{H}$ et al. Is the psoas a hip flexor in the active straight leg raise? Eur Spine J 2011;20(5):759-65.

[50] Mannion AF et al. Spine stabilisation exercises in the treatment of chronic low back pain: a good clinical outcome is not associated with improved abdominal muscle function. Eur Spine J 2012;21(7):1301-10.

[51] Kalichman L et al. Changes in paraspinal muscles and their association with low back pain and spinal degeneration: CT study. Eur Spine J 2010;19(7): $1136-44$.

[52] Mannion AF et al. Spinal segmental stabilisation exercises for chronic low back pain: programme adherence and its influence on clinical outcome. Eur Spine J 2009;18(12):1881-91.

[53] Heneweer $\mathrm{H}$ et al. Physical fitness, rather than self-reported physical activities, is more strongly associated with low back pain: evidence from a working population. Eur Spine J 2012;21(7):1265-72.

[54] Jo HJ et al. A kinematic analysis of relative stability of the lower extremities between subjects with and without chronic low back pain. Eur Spine J 2011;20(8):1297-303.

[55] Barz T et al. Nerve root sedimentation sign: evaluation of a new radiological sign in lumbar spinal stenosis. Spine (Phila Pa 1976) 2010;5(8):892-7.

[56] Barz T et al. Clinical validity of the nerve root sedimentation sign. In: German Spine Congress, Hamburg; 2011.

[57] Deyo RA. Treatment of lumbar spinal stenosis: a balancing act. Spine J 2010;10(7):625-7.

[58] Overdevest GM et al. Design of the Verbiest trial: cost-effectiveness of surgery versus prolonged conservative treatment in patients with lumbar stenosis. BMC Musculoskelet Disord 2011;12:57.

[59] Hansson T, Hansson E, Malchau H. Utility of spine surgery: a comparison of common elective orthopaedic surgical procedures. Spine (Phila Pa 1976) 2008;33(25):2819-30.

[60] Ewen B. Osteopathic procedures and osteopathic medicine. A manual medicine osteopathic career in chain reactions. Manuelle Med 2013;51(4):291-4.

[61] Tsuij H. Comprehensive atlas of lumbar spine surgery. St. Louis: Mosby Year Book; 1990.

[62] Kirkaldy-Willis WH, Bernard TN. Managing low back pain. 4th ed. Oxford: Elsevier; 1999. 